\title{
Applications of a generalized $q$-difference equation
}

Jian-Ping Fang*

\section{"Correspondence:} fjp7402@163.com; fjp7402@sina.com School of Mathematical Science, Huaiyin Normal University, Huaian, Jiangsu 223300, P.R. China

\begin{abstract}
In this paper, we establish one general q-exponential operator identity by solving one simple q-difference equation. Using this q-difference equation, we get some generalizations of Andrews-Askey and Askey-Wilson integral. In addition, we also discuss some properties of q-polynomials $H_{n}$.
\end{abstract}

MSC: 33D05; 33D45; 11B65; 33D60

Keywords: q-difference equation; Andrews-Askey integral; Askey-Wilson integral; q-exponential operator; $q$-polynomials

\section{Introduction and notations}

For decades, various families of $q$-polynomials and $q$-integral have been investigated rather widely and extensively due mainly to their having been found to be potentially useful in such wide variety of fields as theory of partitions, number theory, combinatorial analysis, finite vector spaces, Lie theory, etc. (cf. [1-27]). There are many techniques to achieve the ends; for instance, analysis methods ( $c f .[3-6,14,16])$, combinatorics method (cf. [17]), and $q$-operator method $(c f .[7,9-11,19,24])$ and so on. In resent years, the authors $[8,20-$ $22]$ derived some formulas of $q$-polynomials and $q$-integral from studying the properties of solutions about some $q$-difference equations. Inspired by their work, in this paper, we will present one more generalized $q$-difference equation and give some applications of it.

We adopt the notations used by Gasper and Rahman [15]. Throughout the paper unless otherwise stated we assume that $0<|q|<1$. Let $\mathbb{N}$ denote the set of non-negative integer, $\mathbb{C}$ denote the set of complex numbers.

For any complex number $a$, the $q$-shifted factorial are defined as

$$
(a ; q)_{0}=1, \quad(a ; q)_{n}=\prod_{k=0}^{n-1}\left(1-a q^{k}\right), \quad(a ; q)_{\infty}=\prod_{k=0}^{\infty}\left(1-a q^{k}\right), \quad n \in \mathbb{N}
$$

and we also adopt the following compact notation for the multiple $q$-shifted factorial:

$$
\left(a_{0}, a_{1}, \ldots, a_{m} ; q\right)_{n}=\left(a_{0} ; q\right)_{n}\left(a_{1} ; q\right)_{n} \cdots\left(a_{m} ; q\right)_{n}, \quad m \in \mathbb{N}, n=\infty \text { or } n \in \mathbb{N} .
$$

The basic hypergeometric series ${ }_{s} \Phi_{t}$ is given by

$$
{ }_{s} \Phi_{t}\left(\begin{array}{l}
a_{1}, \ldots, a_{s} \\
b_{1}, \ldots, b_{t}
\end{array} q, x\right)=\sum_{k=0}^{\infty} \frac{\left(a_{1}, a_{2}, \ldots, a_{s} ; q\right)_{k}}{\left(q, b_{1}, \ldots, b_{t} ; q\right)_{k}}\left[(-1)^{k} q^{\left(\frac{k}{2}\right)}\right]^{1+t-s} x^{k}, \quad s, t \in \mathbb{N} .
$$

O2014 Fang; licensee Springer. This is an Open Access article distributed under the terms of the Creative Commons Attribution License (http://creativecommons.org/licenses/by/2.0), which permits unrestricted use, distribution, and reproduction in any medium, provided the original work is properly cited. 
For any function $f(x)$ of one variable, the $q$-derivative of $f(x)$ with respect to $x$ is defined as $(c f .[7-11,18-22])$

$$
D_{q, x}\{f(x)\}=\frac{f(x)-f(q x)}{x},
$$

and we further defined $D_{q, x}^{0}\{f(x)\}=f(x)$, and for $n \geq 1, D_{q, x}^{n}\{f(x)\}=D_{q, x}\left\{D_{q, x}^{n-1}\{f(x)\}\right\}$.

The $q$-binomial coefficient is defined as

$$
\left[\begin{array}{l}
n \\
k
\end{array}\right]= \begin{cases}\frac{(q ; q)_{n}}{(q ; q) \eta_{k}(q ; q)_{n-k}}, & \text { for } 0 \leq k \leq n \\
0, & \text { otherwise. }\end{cases}
$$

For $a_{0}, a_{1}, \ldots, a_{s}, b_{1}, \ldots, b_{s}, b, c \in \mathbb{C}$, we define the following generalized $q$-operator:

$$
F\left(a_{0}, \ldots, a_{s} ; b_{1}, \ldots, b_{s} ; c D_{q, b}\right)={ }_{s+1} \Phi_{s}\left(\begin{array}{c}
a_{0}, a_{1}, \ldots, a_{s} \\
b_{1}, \ldots, b_{s}
\end{array} ; q, c D_{q, b}\right) .
$$

Some special cases of the above $q$-operator had been studied by many researchers. For instance, the authors $[9,19-21,24]$ made a systematic study on $F\left(0 ;-; c D_{q, b}\right)$. Some applications of $F\left(a_{0} ;-; c D_{q, b}\right)$ were given in $[10,11,22]$. Some properties and applications of $F\left(a_{0}, a_{1} ; b_{1} ; c D_{q, b}\right)$ were discussed in $[8,11]$. In this paper, we present the following more generalized $q$-difference equation for the above $q$-operator.

Theorem 1.1 Let $f\left(a_{0}, \ldots, a_{s}, b_{1}, \ldots, b_{s}, b, c\right)$ be $a 2 s+3$-variable analytic function in $a$ neighborhood of $\left(a_{0}, \ldots, a_{s}, b_{1}, \ldots, b_{s}, b, c\right)=(0,0, \ldots, 0) \in \mathbb{C}^{2 s+3}, s \in \mathbb{N}$, satisfying the q-difference equation

$$
\begin{aligned}
& b \sum_{j=0}^{s+1} \frac{(-1)^{j} B_{j}}{q^{j}} f\left(a_{0}, \ldots, b_{1}, \ldots, b_{s}, b, c q^{j}\right)-c \sum_{j=0}^{s+1}(-1)^{j} A_{j}\left[f\left(a_{0}, \ldots, b_{1}, \ldots, b_{s}, b, c q^{j}\right)\right. \\
& \left.\quad-f\left(a_{0}, \ldots, a_{s}, b_{1}, \ldots, b_{s}, b q, c q^{j}\right)\right]=0,
\end{aligned}
$$

where

$$
\begin{aligned}
& b_{0}=q, \quad B_{0}=A_{0}=1, \quad B_{1}=\sum_{i=0}^{s} b_{i}, \quad B_{2}=\sum_{0 \leq i<j \leq s} b_{i} b_{j}, \\
& B_{3}=\sum_{0 \leq i<j<k \leq s} b_{i} b_{j} b_{k}, \ldots, \quad B_{s+1}=b_{0} b_{1} \cdots b_{s}, \quad A_{1}=\sum_{i=0}^{s} a_{i}, \\
& A_{2}=\sum_{0 \leq i<j \leq s} a_{i} a_{j}, \quad A_{3}=\sum_{0 \leq i<j<k \leq s} a_{i} a_{j} a_{k}, \ldots, \quad A_{s+1}=a_{0} a_{1} \cdots a_{s} .
\end{aligned}
$$

Then we have

$$
\begin{aligned}
& f\left(a_{0}, \ldots, a_{s}, b_{1}, \ldots, b_{s}, b, c\right) \\
& \quad=F\left(a_{0}, \ldots, a_{s} ; b_{1}, \ldots, b_{s} ; c D_{q, b}\right) f\left(a_{0}, \ldots, a_{s}, b_{1}, \ldots, b_{s}, b, 0\right) .
\end{aligned}
$$


Corollary 1.2 ([8], Eq. (1.12)) Let $f\left(a_{0}, a_{1}, b_{1}, b, c\right)$ be a 5-variable analytic function in a neighborhood of $\left(a_{0}, a_{1}, b_{1}, b, c\right)=(0,0,0,0,0) \in \mathbb{C}^{5}$, satisfying the q-difference equation

$$
\begin{aligned}
& b\left[f\left(a_{0}, a_{1}, b_{1}, b, c\right)-\left(1+q^{-1} b_{1}\right) f\left(a_{0}, a_{1}, b_{1}, b, c q\right)+q^{-1} b_{1} f\left(a_{0}, a_{1}, b_{1}, b, c q^{2}\right)\right] \\
& \quad-c\left[\left(f\left(a_{0}, a_{1}, b_{1}, b, c\right)-f\left(a_{0}, a_{1}, b_{1}, b q, c\right)\right)-\left(a_{0}+a_{1}\right)\left(f\left(a_{0}, a_{1}, b_{1}, b, c q\right)\right.\right. \\
& \left.\left.\quad-f\left(a_{0}, a_{1}, b_{1}, b q, c q\right)\right)-a_{0} a_{1}\left(f\left(a_{0}, a_{1}, b_{1}, b, c q^{2}\right)-f\left(a_{0}, a_{1}, b_{1}, b q, c q^{2}\right)\right)\right]=0,
\end{aligned}
$$

then

$$
f\left(a_{0}, a_{1}, b_{1}, b, c\right)=F\left(a_{0}, a_{1} ; b_{1} ; c D_{q, b}\right) f\left(a_{0}, a_{1}, b_{1}, b, 0\right) .
$$

Remark 1.3 Letting $a_{i}=b_{i}=0, i=2,3, \ldots, s$, Eq. (6) reduces to (9). Setting $a_{i}=b_{i}=0$, $i=0,1, \ldots, s$, then replacing $b, c$ by $a, b$ respectively, Eq. (6) reduces to [20], Theorem 1 . Putting $a_{i}=b_{i}=0, i=1,2, \ldots, s$, then replacing $a_{0}, b, c$ by $a, c, b$, respectively, Eq. (6) reduces to [22], Proposition 1.2.

Proof of Theorem 1.1 From the theory of several complex variables in [28] (or [25], p.28, Hartog's theorem), we assume that

$$
f\left(a_{0}, \ldots, a_{s}, b_{1}, \ldots, b_{s}, b, c\right)=\sum_{n=0}^{\infty} W_{n}\left(a_{0}, \ldots, a_{s}, b_{1}, \ldots, b_{s}, b\right) c^{n}
$$

and then substitute the above equation into (6) yielding

$$
\begin{aligned}
b \sum_{j=0}^{s+1} \frac{(-1)^{j} B_{j}}{q^{j}} \sum_{n=0}^{\infty} W_{n}\left(a_{0}, \ldots, a_{s}, b_{1}, \ldots, b_{s}, b\right) c^{n} q^{j n} \\
=c \sum_{j=0}^{s+1}(-1)^{j} A_{j}\left[\sum_{n=0}^{\infty} W_{n}\left(a_{0}, \ldots, a_{s}, b_{1}, \ldots, b_{s}, b\right) c^{n} q^{j n}\right. \\
\left.\quad-\sum_{n=0}^{\infty} W_{n}\left(a_{0}, \ldots, a_{s}, b_{1}, \ldots, b_{s}, b q\right) c^{n} q^{j n}\right]
\end{aligned}
$$

Equating the coefficients of $c^{n}$, we have

$$
\begin{aligned}
& b \sum_{j=0}^{s+1}(-1)^{j} B_{j} q^{j(n-1)} W_{n}\left(a_{0}, \ldots, a_{s}, b_{1}, \ldots, b_{s}, b\right) \\
& =\sum_{j=0}^{s+1}(-1)^{j} q^{j(n-1)} A_{j}\left[W_{n-1}\left(a_{0}, \ldots, a_{s}, b_{1}, \ldots, b_{s}, b\right)\right. \\
& \left.\quad-W_{n-1}\left(a_{0}, \ldots, a_{s}, b_{1}, \ldots, b_{s}, b q\right)\right] .
\end{aligned}
$$

For each $n \geq 1$, we get

$$
\begin{aligned}
& W_{n}\left(a_{0}, \ldots, a_{s}, b_{1}, \ldots, b_{s}, b\right) \\
& \quad=\frac{\left(1-a_{0} q^{n-1}\right)\left(1-a_{1} q^{n-1}\right) \cdots\left(1-a_{s} q^{n-1}\right)}{\left(1-b_{0} q^{n-1}\right)\left(1-b_{1} q^{n-1}\right) \cdots\left(1-b_{s} q^{n-1}\right)} D_{q, b}\left\{W_{n-1}\left(a_{0}, \ldots, a_{s}, b_{1}, \ldots, b_{s}, b\right)\right\} .
\end{aligned}
$$


By iteration, we find that

$$
\begin{aligned}
& W_{n}\left(a_{0}, \ldots, a_{s}, b_{1}, \ldots, b_{s}, b\right) \\
& \quad=\frac{\left(a_{0} ; q\right)_{n}\left(a_{1} ; q\right)_{n} \cdots\left(a_{s} ; q\right)_{n}}{(q ; q)_{n}\left(b_{1} ; q\right)_{n} \cdots\left(b_{s} ; q\right)_{n}} D_{q, b}^{n}\left\{W_{0}\left(a_{0}, \ldots, a_{s}, b_{1}, \ldots, b_{s}, b\right)\right\} .
\end{aligned}
$$

Putting $c=0$ in (11), we get $W_{0}\left(a_{0}, \ldots, a_{s}, b_{1}, \ldots, b_{s}, b\right)=f\left(a_{0}, \ldots, a_{s}, b_{1}, \ldots, b_{s}, b, 0\right)$. Substituting (15) into (11), we get (8). This completes the proof.

Theorem 1.4 If $a_{0}=q^{-G}, G \in \mathbb{N}, b, w, u, v, a_{i}, b_{i} \in \mathbb{C}, i=1,2, \ldots, s$, then

$$
\begin{aligned}
& F\left(a_{0}, \ldots, a_{s} ; b_{1}, \ldots, b_{s} ; c D_{q, b}\right)\left\{\frac{(b w ; q)_{\infty}}{(b u, b v ; q)_{\infty}}\right\} \\
& \quad=\frac{(b w ; q)_{\infty}}{(b u, b v ; q)_{\infty}} \sum_{n=0}^{\infty} \frac{\left(a_{0}, a_{1}, \ldots, a_{s} ; q\right)_{n}}{\left(q, b_{1}, \ldots, b_{s} ; q\right)_{n}}\left(\frac{c}{b}\right)^{n}{ }_{3} \Phi_{2}\left(\begin{array}{r}
q^{-n}, b u, b v \\
0, b w
\end{array} q, q\right) .
\end{aligned}
$$

Proof We use $f\left(a_{0}, \ldots, a_{s}, b_{1}, \ldots, b_{s}, b, c\right)$ to denote the right side of (16). We have

$$
W_{n}=\frac{\left(a_{0}, a_{1}, \ldots, a_{s} ; q\right)_{n}}{\left(q, b_{1}, \ldots, b_{s} ; q\right)_{n}}
$$

and $A_{i}, B_{i}(i=0,1, \ldots, s)$ are defined as (7), we have

$$
\begin{aligned}
& b \sum_{j=0}^{s+1} \frac{(-1)^{j} B_{j}}{q^{j}} f\left(a_{0}, \ldots, b_{1}, \ldots, b_{s}, b, c q^{j}\right) \\
& =b \frac{(b w ; q)_{\infty}}{(b u, v ; q)_{\infty}} \sum_{n=0}^{\infty} W_{n}\left(\frac{c}{b}\right)^{n} \sum_{k=0}^{n}\left[\begin{array}{l}
n \\
k
\end{array}\right] \\
& \times \frac{(b u, b v ; q)_{k}}{(b w ; q)_{k}}(-1)^{k} q^{\left(\begin{array}{c}
k \\
2
\end{array}\right)+k-n k}\left[1-B_{1} q^{n-1}+\cdots+(-1)^{s+1} B_{s+1} q^{(s+1)(n-1)}\right] \\
& =\frac{b(b w q ; q)_{\infty}}{(b u q, b v q ; q)_{\infty}} \sum_{n=0}^{\infty} W_{n}\left(\frac{c}{b}\right)^{n} \sum_{k=0}^{n}\left(\left[\begin{array}{c}
n-1 \\
k
\end{array}\right] q^{k}+\left[\begin{array}{c}
n-1 \\
k-1
\end{array}\right]\right) \\
& \times \frac{(b u q, b v q ; q)_{k-1}}{(b w q ; q)_{k-1}}(-1)^{k} q^{\left(\begin{array}{c}
k \\
2
\end{array}\right)+k-n k}\left(1-b_{0} q^{n-1}\right) \cdots\left(1-b_{s} q^{n-1}\right) \\
& =\frac{c(b w q ; q)_{\infty}}{(b u q, b v q ; q)_{\infty}} \sum_{n=1}^{\infty} W_{n-1}\left(\frac{c}{b}\right)^{n-1} \sum_{k=0}^{n}\left[\begin{array}{c}
n-1 \\
k
\end{array}\right] \frac{(b u q, b v q ; q)_{k-1}}{(b w q ; q)_{k-1}}(-1)^{k} q^{\left(\begin{array}{l}
k \\
2
\end{array}\right)+2 k-n k} \\
& \times\left(1-a_{0} q^{n-1}\right) \cdots\left(1-a_{s} q^{n-1}\right)-\frac{c(b w q ; q)_{\infty}}{(b u q, b v q ; q)_{\infty}} \sum_{n=1}^{\infty} W_{n-1}\left(\frac{c}{b}\right)^{n-1} \sum_{k=0}^{n}\left[\begin{array}{l}
n-1 \\
k-1
\end{array}\right] \\
& \times \frac{(b u q, b v q ; q)_{k-1}}{(b w q ; q)_{k-1}}(-1)^{k-1} q^{\left(\begin{array}{c}
k \\
2
\end{array}\right)+k-n k}\left(1-a_{0} q^{n-1}\right) \cdots\left(1-a_{s} q^{n-1}\right) \text {. }
\end{aligned}
$$

Replacing $n-1$ by $n$, then applying (4), we find that the above equation is equal to

$$
\begin{aligned}
& \frac{c(b w ; q)_{\infty}}{(b u, b v ; q)_{\infty}} \sum_{n=0}^{\infty} W_{n}\left(\frac{c}{b}\right)^{n} \sum_{k=0}^{n+1}\left[\begin{array}{l}
n \\
k
\end{array}\right] \frac{(b u, b v ; q)_{k}}{(b w ; q)_{k}}(-1)^{k} q^{\left(\begin{array}{c}
k \\
2
\end{array}\right)+k-n k}\left(1-a_{0} q^{n}\right) \cdots\left(1-a_{s} q^{n}\right) \\
& \quad-\frac{c(b w q ; q)_{\infty}}{(b u q, b v q ; q)_{\infty}} \sum_{n=0}^{\infty} W_{n}\left(\frac{c}{b q}\right)^{n} \sum_{k=0}^{n+1}\left[\begin{array}{l}
n \\
k
\end{array}\right]
\end{aligned}
$$




$$
\begin{aligned}
& \times \frac{(b u q, b v q ; q)_{k}}{(b w q ; q)_{k}}(-1)^{k} q^{\left(\frac{k}{2}\right)+k-n k}\left(1-a_{0} q^{n}\right) \cdots\left(1-a_{s} q^{n}\right) \\
= & \frac{c(b w ; q)_{\infty}}{(b u, b v ; q)_{\infty}} \sum_{n=0}^{\infty} W_{n}\left(\frac{c}{b}\right)^{n} \sum_{k=0}^{n}\left[\begin{array}{l}
n \\
k
\end{array}\right] \\
& \times \frac{(b u, b v ; q)_{k}}{(b w ; q)_{k}}(-1)^{k} q^{\left(\frac{k}{2}\right)+k-n k}\left(1-a_{0} q^{n}\right) \cdots\left(1-a_{s} q^{n}\right) \\
& -\frac{c(b w q ; q)_{\infty}}{(b u q, b v q ; q)_{\infty}} \sum_{n=0}^{\infty} W_{n}\left(\frac{c}{b q}\right)^{n} \sum_{k=0}^{n}\left[\begin{array}{l}
n \\
k
\end{array}\right] \\
& \times \frac{(b u q, b v q ; q)_{k}}{(b w q ; q)_{k}}(-1)^{k} q^{\left(\begin{array}{l}
k \\
2
\end{array}\right)+k-n k}\left(1-a_{0} q^{n}\right) \cdots\left(1-a_{s} q^{n}\right) \\
= & c \sum_{j=0}^{s+1}(-1)^{j} A_{j}\left[f\left(a_{0}, \ldots, b_{1}, \ldots, b_{s}, b, c q^{j}\right)-f\left(a_{0}, \ldots, a_{s}, b_{1}, \ldots, b_{s}, b q, c q^{j}\right)\right] .
\end{aligned}
$$

So $f\left(a_{0}, \ldots, a_{s}, b_{1}, \ldots, b_{s}, b, c\right)$ satisfies (6), applying (8), we complete the proof.

Letting $e \rightarrow 0, b \rightarrow b u, c \rightarrow b v, d \rightarrow b w$ in Eq. (III.12) ([15], p.360), we have

$$
\left(\frac{1}{b}\right)^{n}{ }_{3} \Phi_{2}\left(\begin{array}{r}
q^{-n}, b u, b v \\
0, b w
\end{array} ;, q\right)=v^{n}{ }_{3} \Phi_{1}\left(\begin{array}{r}
q^{-n}, w / u, b v \\
b w
\end{array} ;, u q^{n} / v\right) .
$$

Combining the above identity and (16), we find the following generalized formula of [11], Lemma 2.3 (or [8], Eq. (3.4)).

Corollary 1.5 If $a_{0}=q^{-G}, G \in \mathbb{N}, b, w, u, v, a_{i}, b_{i} \in \mathbb{C}, i=1,2, \ldots, s$, then

$$
\begin{aligned}
& F\left(a_{0}, \ldots, a_{s} ; b_{1}, \ldots, b_{s} ; c D_{q, b}\right)\left\{\frac{(b w ; q)_{\infty}}{(b u, b v ; q)_{\infty}}\right\} \\
& \quad=\frac{(b w ; q)_{\infty}}{(b u, b v ; q)_{\infty}} \sum_{n=0}^{\infty} \frac{\left(a_{0}, a_{1}, \ldots, a_{s} ; q\right)_{n}}{\left(q, b_{1}, \ldots, b_{s} ; q\right)_{n}}(c v)^{n}{ }_{3} \Phi_{1}\left(\begin{array}{r}
q^{-n}, w / u, b v \\
b w
\end{array} q, u q^{n} / v\right) .
\end{aligned}
$$

Letting $w=0$ in (16), we find the following.

Corollary 1.6 If $a_{0}=q^{-G}, G \in \mathbb{N}, b, c, u, v, a_{i}, b_{i} \in \mathbb{C}, i=1,2, \ldots, s$, then

$$
\begin{aligned}
& F\left(a_{0}, \ldots, a_{s} ; b_{1}, \ldots, b_{s} ; c D_{q, b}\right)\left\{\frac{1}{(b u, b v ; q)_{\infty}}\right\} \\
& \quad=\frac{1}{(b u, b v ; q)_{\infty}} \sum_{n=0}^{\infty} \frac{\left(a_{0}, a_{1}, \ldots, a_{s} ; q\right)_{n}}{\left(q, b_{1}, \ldots, b_{s} ; q\right)_{n}}\left(\frac{c}{b}\right)^{n}{ }_{3} \Phi_{2}\left(\begin{array}{r}
q^{-n}, b u, b v \\
0,0
\end{array} q, q\right) .
\end{aligned}
$$

Letting $w=v=0$ in (16), we find the following.

Corollary 1.7 If $\max \{|b u|,|c u|\}<1, u, a_{i}, b_{i} \in \mathbb{C}, i=1,2, \ldots, s$, then

$$
F\left(a_{0}, \ldots, a_{s} ; b_{1}, \ldots, b_{s} ; c D_{q, b}\right)\left\{\frac{1}{(b u ; q)_{\infty}}\right\}=\frac{1}{(b u ; q)_{\infty}} \sum_{n=0}^{\infty} \frac{\left(a_{0}, a_{1}, \ldots, a_{s} ; q\right)_{n}}{\left(q, b_{1}, \ldots, b_{s} ; q\right)_{n}}(c u)^{n} .
$$


Letting $v=0$ in (16), then applying $q$-Chu-Vandermonde summation ([15], p.354, Eq. (II.6))

$$
{ }_{2} \Phi_{1}\left(\begin{array}{c}
q^{-n}, a \\
c
\end{array} ;, q\right)=\frac{(c / a ; q)_{n}}{(c ; q)_{n}} a^{n}
$$

and we obtain the following.

Corollary 1.8 If $\max \{|b u|,|b w|,|c u|\}<1, u, v, a_{i}, b_{i} \in \mathbb{C}, i=1,2, \ldots, s$, then

$$
\begin{aligned}
& F\left(a_{0}, \ldots, a_{s} ; b_{1}, \ldots, b_{s} ; c D_{q, b}\right)\left\{\frac{(b w ; q)_{\infty}}{(b u ; q)_{\infty}}\right\} \\
& \quad=\frac{(b w ; q)_{\infty}}{(b u ; q)_{\infty}} \sum_{n=0}^{\infty} \frac{\left(a_{0}, a_{1}, \ldots, a_{s}, w / u ; q\right)_{n}}{\left(q, b_{1}, \ldots, b_{s}, b w ; q\right)_{n}}(c u)^{n} .
\end{aligned}
$$

Remark 1.9 It were difficult to distinguish analysis of the functions of the right side of (16) (or (21), (22)) if we would remove the condition $a_{0}=q^{-G}$. But in (23) and (25), we do not need the condition $a_{0}=q^{-G}$. Under $\max \{|b u|,|b v|,|b w|,|c u|\}<1$, it is easy to verify that the right sides of (23) and (25) are analytic functions in a neighborhood of $(0,0, \ldots, 0) \in \mathbb{C}^{2 s+3}$. In this paper, the symbols $W_{n}$ and $U_{n}$ are frequently used. Here $W_{n}$ is defined as (17), and $U_{n}$ is equal to $\left(h_{0}, h_{1}, \ldots, h_{t} ; q\right)_{n} /\left(q, g_{1}, \ldots, g_{t} ; q\right)_{n}$.

The paper is organized in the following manner. In the next two sections we give some generalizations of Andrews-Askey and Askey-Wilson integrals by the $q$-difference equation. In Section 4, we discuss some properties of $q$-polynomials $H_{n}$. Several special cases and examples of our results are also pointed out, in the concluding section.

\section{Generalizations of Andrews-Askey integrals}

We have

$$
\int_{c}^{d} \frac{(q x / c, q x / d ; q)_{\infty}}{(a x, b x ; q)_{\infty}} d_{q} x=\frac{d(1-q)(q, d q / c, c / d, a b c d ; q)_{\infty}}{(a c, a d, b c, b d ; q)_{\infty}}
$$

provided that there are no zero factors in the denominator of the integral, which could be directly derived from Andrews-Askey integrals ([3], Eq. (2.1) or [5], Eq. (1.15)) after some simple replacing. In [8] (or [21, 26, 27]), some generalizations and applications of (26) are given. In this paper we give the following generalizations of the above identity.

Theorem 2.1 If $a_{0}=q^{-N}, a_{i}, b_{i} \in \mathbb{C}, i=1,2, \ldots, s, N \in \mathbb{N}$, then

$$
\begin{gathered}
\int_{c}^{d} \frac{(q x / c, q x / d ; q)_{\infty}}{(a x, b x ; q)_{\infty}} \sum_{n=0}^{\infty} W_{n}\left(\frac{e}{a}\right)^{n}{ }_{3} \Phi_{2}\left(\begin{array}{r}
q^{-n}, a x, a b c d \\
0, a c
\end{array} ; q, q\right) d_{q} x \\
\quad=\frac{d(1-q)(q, d q / c, c / d, a b c d ; q)_{\infty}}{(a c, a d, b c, b d ; q)_{\infty}} \sum_{n=0}^{\infty} W_{n}(d e)^{n} .
\end{gathered}
$$

Applying (20), we rewrite (27) as follows. 
Corollary 2.2 If $a_{0}=q^{-N}, a_{i}, b_{i} \in \mathbb{C}, i=1,2, \ldots, s, N \in \mathbb{N}$, then

$$
\begin{aligned}
& \int_{c}^{d} \frac{(q x / c, q x / d ; q)_{\infty}}{(a x, b x ; q)_{\infty}} \sum_{n=0}^{\infty} W_{n}(b c d e)^{n}{ }_{3} \Phi_{1}\left(\begin{array}{r}
q^{-n}, c / x, a b c d \\
a c
\end{array} q, x q^{n} / b c d\right) d_{q^{x}} \\
& =\frac{d(1-q)(q, d q / c, c / d, a b c d ; q)_{\infty}}{(a c, a d, b c, b d ; q)_{\infty}} \sum_{n=0}^{\infty} W_{n}(d e)^{n} .
\end{aligned}
$$

If $a_{i}=b_{i}=0, i=2,3, \ldots, s$, then letting $e=q / b c d$, the left-hand side of (28) is equal to

$$
\begin{aligned}
& \int_{c}^{d} \frac{(q x / c, q x / d ; q)_{\infty}}{(a x, b x ; q)_{\infty}} \sum_{n=0}^{\infty} \frac{\left(a_{0}, a_{1} ; q\right)_{n}}{\left(q, b_{1} ; q\right)_{n}} q^{n}{ }_{3} \Phi_{1}\left(q^{-n}, c / x, a b c d ; q, x q^{n} / b c d\right) d_{q} x \\
& =\int_{c}^{d} \frac{(q x / c, q x / d ; q)_{\infty}}{(a x, b x ; q)_{\infty}} \sum_{k=0}^{\infty} \frac{\left(a_{0}, a_{1}, c / x, a b c d ; q\right)_{k}}{\left(q, a c, b_{1} ; q\right)_{k}} \\
& \quad \times\left(\frac{q x}{b c d}\right)^{k} \sum_{n=0}^{\infty} \frac{\left(a_{0} q^{k}, a_{1} q^{k} ; q\right)_{n} q^{n}}{\left(q, b_{1} q^{k} ; q\right)_{n}} d_{q} x .
\end{aligned}
$$

For $a_{0}=q^{-N}$, the inner summation is equal to

$$
\frac{\left(b_{1} / a_{1} ; q\right)_{N-k}\left(a_{1} q^{k}\right)^{N-k}}{\left(b_{1} q^{k} ; q\right)_{N-k}}=(-1)^{k} q^{-\left(\begin{array}{c}
k \\
2
\end{array}\right)} a_{1}^{N} \frac{\left(b_{1} ; q\right)_{k} b_{1}^{-k}}{\left(q a_{0} a_{1} / b_{1} ; q\right)_{k}} \frac{\left(b_{1} / a_{1} ; q\right)_{N}}{\left(b_{1} ; q\right)_{N}} .
$$

Substituting the above identity into (29), we have the following.

Corollary 2.3 ([8], Theorem 14) If $a_{0}=q^{-N}, a_{1}, b_{1} \in \mathbb{C}, N \in \mathbb{N}$, then

$$
\begin{aligned}
& \int_{c}^{d} \frac{(q x / c, q x / d ; q)_{\infty}}{(a x, b x ; q)_{\infty}}{ }_{4} \Phi_{2}\left(\begin{array}{c}
a_{0}, a_{1}, c / x, a b c d \\
a c, q a_{0} a_{1} / b_{1}
\end{array} ; q, x q / b c d\right) d_{q} x \\
& \quad=\frac{d(1-q)(q, d q / c, c / d, a b c d ; q)_{\infty}\left(b_{1} ; q\right)_{N}}{(a c, a d, b c, b d ; q)_{\infty}\left(b_{1} / a_{1} ; q\right)_{N} a_{1}^{N}} \Phi_{1}\left(\begin{array}{c}
a_{0}, a_{1} \\
b_{1}
\end{array} q, q / b c\right)
\end{aligned}
$$

Remark 2.4 For $a_{0}=q^{-N}$, we find that

$$
\frac{\left(b_{1} ; q\right)_{N}}{\left(b_{1} / a_{1} ; q\right)_{N} a_{1}^{N}}=\frac{\left(q a_{1} / b_{1}, q a_{0} / b_{1} ; q\right)_{\infty}}{\left(q a_{0} a_{1} / b_{1}, q / b_{1} ; q\right)_{\infty}} .
$$

So we see that the identity (31) is the same as Theorem 14 in [8] after replacing $\left(a_{0}, a_{1}, b_{1}\right)$ by $(r, w, v)$, respectively.

Proof of Theorem 2.1 We rewrite (27) as follows:

$$
\begin{aligned}
& \int_{c}^{d} \frac{(q x / c, q x / d ; q)_{\infty}}{(b x ; q)_{\infty}} \frac{(a c ; q)_{\infty}}{(a x, a b c d ; q)_{\infty}} \sum_{n=0}^{\infty} W_{n}\left(\frac{e}{a}\right)^{n}{ }_{3} \Phi_{2}\left(\begin{array}{r}
q^{-n}, a x, a b c d \\
0, a c
\end{array} ;, q\right) d_{q} x \\
& =\frac{d(1-q)(q, d q / c, c / d ; q)_{\infty}}{(b c, b d ; q)_{\infty}} \frac{1}{(a d ; q)_{\infty}} \sum_{n=0}^{\infty} W_{n}(d e)^{n} .
\end{aligned}
$$


If we use $f_{L}=f_{L}\left(a_{0}, \ldots, a_{s}, b_{1}, \ldots, b_{s}, a, e\right)$ we have

$$
\frac{(a c ; q)_{\infty}}{(a x, a b c d ; q)_{\infty}} \sum_{n=0}^{\infty} W_{n}\left(\frac{e}{a}\right)^{n}{ }_{3} \Phi_{2}\left(\begin{array}{r}
q^{-n}, a x, a b c d \\
0, a c
\end{array} ;, q\right)
$$

In the same way as proving (16), we can verify $f_{L}$ satisfies $(6)$, so we have

$$
\begin{aligned}
f_{L} & =F\left(a_{0}, \ldots, a_{s} ; b_{1}, \ldots, b_{s}, e D_{q, a}\right)\left\{f_{L}\left(a_{0}, \ldots, a_{s}, b_{1}, \ldots, b_{s}, a, 0\right)\right\} \\
& =F\left(a_{0}, \ldots, a_{s} ; b_{1}, \ldots, b_{s}, e D_{q, a}\right)\left\{\frac{(a c ; q)_{\infty}}{(a x, a b c d ; q)_{\infty}}\right\} .
\end{aligned}
$$

We use $f_{R}=f_{R}\left(a_{0}, \ldots, a_{s}, b_{1}, \ldots, b_{s}, a, e\right)$ and we have

$$
\frac{1}{(a d ; q)_{\infty}} \sum_{n=0}^{\infty} W_{n}(d e)^{n}
$$

It is easy to prove $f_{R}$ satisfies (6), so we find that

$$
\begin{aligned}
f_{R} & =F\left(a_{0}, \ldots, a_{s} ; b_{1}, \ldots, b_{s}, e D_{q, a}\right)\left\{f_{R}\left(a_{0}, \ldots, a_{s}, b_{1}, \ldots, b_{s}, a, 0\right)\right\} \\
& =F\left(a_{0}, \ldots, a_{s} ; b_{1}, \ldots, b_{s}, e D_{q, a}\right)\left\{\frac{1}{(a d ; q)_{\infty}}\right\} .
\end{aligned}
$$

Combining the above identity and (26), we complete the proof of (27).

Theorem 2.5 If $a_{0}, a_{i}, b_{i} \in \mathbb{C}, \max \{|a x|,|e x|,|a c|,|a b c d|,|a d|,|d e|\}<1, i=1,2, \ldots, s, N \in \mathbb{N}$, then

$$
\begin{aligned}
& \int_{c}^{d} \frac{(q x / c, q x / d ; q)_{\infty}}{(a x, b x ; q)_{\infty}} \sum_{n=0}^{\infty} \frac{\left(a_{0}, a_{1}, \ldots, a_{s}, c / x ; q\right)_{n}}{\left(q, b_{1}, \ldots, b_{s}, a c ; q\right)_{n}}(e x)^{n} d_{q} x \\
& \quad=\frac{d(1-q)(q, d q / c, c / d, a b c d ; q)_{\infty}}{(a c, a d, b c, b d ; q)_{\infty}} \sum_{n=0}^{\infty} \frac{\left(a_{0}, a_{1}, \ldots, a_{s}, b c ; q\right)_{n}}{\left(q, b_{1}, \ldots, b_{s}, a b c d ; q\right)_{n}}(d e)^{n}
\end{aligned}
$$

Proof We rewrite (38) as follows:

$$
\begin{gathered}
\int_{c}^{d} \frac{(q x / c, q x / d ; q)_{\infty}}{(b x ; q)_{\infty}} \frac{(a c ; q)_{\infty}}{(a x ; q)_{\infty}} \sum_{n=0}^{\infty} \frac{\left(a_{0}, a_{1}, \ldots, a_{s}, c / x ; q\right)_{n}}{\left(q, b_{1}, \ldots, b_{s}, a c ; q\right)_{n}}(e x)^{n} d_{q} x \\
=\frac{d(1-q)(q, d q / c, c / d ; q)_{\infty}}{(b c, b d ; q)_{\infty}} \frac{(a b c d ; q)_{\infty}}{(a d ; q)_{\infty}} \sum_{n=0}^{\infty} \frac{\left(a_{0}, a_{1}, \ldots, a_{s}, b c ; q\right)_{n}}{\left(q, b_{1}, \ldots, b_{s}, a b c d ; q\right)_{n}}(d e)^{n}
\end{gathered}
$$

Setting $f_{L}=f_{L}\left(a_{0}, a_{1}, \ldots, a_{s}, b_{1}, \ldots, b_{s}, a, e\right)$ and $f_{R}=f_{R}\left(a_{0}, a_{1}, \ldots, a_{s}, b_{1}, \ldots, b_{s}, a, e\right)$ denoting the left-hand and the right-hand side of (39), respectively, and taking $v=0$ in proving of Theorem 1.4, we can verify both $f_{L}$ and $f_{R}$ satisfy (6). Letting $F=F\left(a_{0}, a_{1}, \ldots, a_{s} ; b_{1}, \ldots, b_{s}\right.$, $\left.e D_{q, a}\right)$, from (25), we get

$$
\begin{aligned}
f_{L} & =F\left\{f_{L}\left(a_{0}, a_{1}, \ldots, a_{s}, b_{1}, \ldots, b_{s}, a, 0\right)\right\} \\
& =F\left\{\int_{c}^{d} \frac{(q x / c, q x / d ; q)_{\infty}}{(b x ; q)_{\infty}} \frac{(a c ; q)_{\infty}}{(a x ; q)_{\infty}} d_{q} x\right\}
\end{aligned}
$$




$$
\begin{aligned}
& =F\left\{\frac{d(1-q)(q, d q / c, c / d ; q)_{\infty}}{(b c, b d ; q)_{\infty}} \frac{(a b c d ; q)_{\infty}}{(a d ; q)_{\infty}}\right\} \\
& =F\left\{f_{R}\left(a_{0}, a_{1}, \ldots, a_{s}, b_{1}, \ldots, b_{s}, a, 0\right)\right\}=f_{R} .
\end{aligned}
$$

This completes the proof.

Theorem 2.6 If $a_{0}=q^{-N}, a_{i}, b_{i} \in \mathbb{C}, i=1,2, \ldots, s, s, N \in \mathbb{N}$, then

$$
\begin{aligned}
& \int_{c}^{d} \frac{(q x / c, q x / d ; q)_{\infty}}{(a x, b x ; q)_{\infty}}{ }_{s+1} \Phi_{s}\left(\begin{array}{c}
a_{0}, a_{1}, \ldots, a_{s} \\
b_{1}, \ldots, b_{s}
\end{array} ;, e x\right) d_{q} x \\
& \quad=\frac{d(1-q)(q, d q / c, c / d, a b c d ; q)_{\infty}}{(a c, a d, b c, b d ; q)_{\infty}} \sum_{n=0}^{\infty} W_{n}\left(\frac{e}{a}\right)^{n}{ }_{3} \Phi_{2}\left(\begin{array}{r}
q^{-n}, a c, a d \\
0, a b c d
\end{array} q, q\right) .
\end{aligned}
$$

Proof Letting

$$
f_{L}=f_{L}\left(a_{0}, \ldots, a_{s}, b_{1}, \ldots, b_{s}, a, e\right)=\frac{1}{(a x ; q)_{\infty}}{ }_{s+1} \Phi_{s}\left(\begin{array}{c}
a_{0}, a_{1}, \ldots, a_{s} \\
b_{1}, \ldots, b_{s}
\end{array} ; q, e x\right)
$$

and

$$
\begin{aligned}
f_{R} & =f_{R}\left(a_{0}, \ldots, a_{s}, b_{1}, \ldots, b_{s}, a, e\right) \\
& =\frac{(a b c d ; q)_{\infty}}{(a c, a d ; q)_{\infty}} \sum_{n=0}^{\infty} W_{n}\left(\frac{e}{a}\right)^{n}{ }_{3} \Phi_{2}\left(\begin{array}{c}
q^{-n}, a c, a d \\
0, a b c d
\end{array} ;, q\right)
\end{aligned}
$$

we can easily verify both of the above identities satisfy (6), so we have

$$
\begin{aligned}
f_{L} & =F\left(a_{0}, \ldots, a_{s} ; b_{1}, \ldots, b_{s}, e D_{q, a}\right)\left\{f_{L}\left(a_{0}, \ldots, a_{s}, b_{1}, \ldots, b_{s}, a, 0\right)\right\} \\
& =F\left(a_{0}, \ldots, a_{s} ; b_{1}, \ldots, b_{s}, e D_{q, a}\right)\left\{\frac{1}{(a x ; q)_{\infty}}\right\}
\end{aligned}
$$

and

$$
\begin{aligned}
f_{R} & =F\left(a_{0}, \ldots, a_{s} ; b_{1}, \ldots, b_{s}, e D_{q, a}\right)\left\{f_{R}\left(a_{0}, \ldots, a_{s}, b_{1}, \ldots, b_{s}, a, 0\right)\right\} \\
& =F\left(a_{0}, \ldots, a_{s} ; b_{1}, \ldots, b_{s}, e D_{q, a}\right)\left\{\frac{(a b c d ; q)_{\infty}}{(a c, a d ; q)_{\infty}}\right\}
\end{aligned}
$$

Combining (26), we complete the proof of (41).

Interchanging $a$ and $b$ in (41), we get

$$
\begin{aligned}
& \int_{c}^{d} \frac{(q x / c, q x / d ; q)_{\infty}}{(a x, b x ; q)_{\infty}}{ }_{s+1} \Phi_{s}\left(\begin{array}{c}
a_{0}, a_{1}, \ldots, a_{s} \\
b_{1}, \ldots, b_{s}
\end{array} ;, e x\right) d_{q} x \\
& \quad=\frac{d(1-q)(q, d q / c, c / d, a b c d ; q)_{\infty}}{(a c, a d, b c, b d ; q)_{\infty}} \sum_{n=0}^{\infty} W_{n}\left(\frac{e}{b}\right)^{n}{ }_{3} \Phi_{2}\left(\begin{array}{c}
q^{-n}, b c, b d \\
0, a b c d
\end{array} ; q, q\right) .
\end{aligned}
$$

Combing the above identity and (41), then replacing $(b c, b d, a b c d)$ by $(b, c, d)$, respectively, we recover the special case for $e=0$ in Eq. (III.11) ([15], p.360). 
Corollary 2.7 ([15], p.360, Eq. (III.11)) We have

$$
{ }_{3} \Phi_{2}\left(\begin{array}{r}
q^{-n}, b, c \\
0, d
\end{array} ; q, q\right)=\left(\frac{b c}{d}\right)_{3}^{n} \Phi_{2}\left(\begin{array}{r}
q^{-n}, d / b, d / c \\
0, d
\end{array} ;, q\right) .
$$

Theorem 2.8 If $a_{0}=q^{-N}, h_{0}=q^{-G}, a_{i}, b_{i}, h_{j}, g_{j} \in \mathbb{C}, i=1,2, \ldots, s, j=1,2, \ldots, t, s, t, G, N \in \mathbb{N}$, then

$$
\begin{aligned}
\int_{c}^{d} \frac{(q x / c, q x / d ; q)_{\infty}}{(a x, b x ; q)_{\infty}}{ }_{s+1} \Phi_{s}\left(\begin{array}{c}
a_{0}, a_{1}, \ldots, a_{s} \\
b_{1}, \ldots, b_{s}
\end{array} ;, e x\right){ }_{t+1} \Phi_{t}\left(\begin{array}{c}
h_{0}, h_{1}, \ldots, h_{t} \\
g_{1}, \ldots, g_{t}
\end{array} q, f x\right) d_{q} x \\
=\frac{d(1-q)(q, d q / c, c / d, a b c d ; q)_{\infty}}{(a c, a d, b c, b d ; q)_{\infty}} \\
\quad \times \sum_{n=0}^{\infty} \sum_{l=0}^{\infty} \sum_{k=0}^{n} \sum_{j=0}^{l} W_{n} U_{l}\left(\frac{e}{a}\right)^{n}\left(\frac{f}{b}\right)^{l} \frac{\left(q^{-n}, a c, a d ; q\right)_{k}}{(q, a b c d ; q)_{k}} \frac{\left(q^{-l}, b c, b d ; q\right)_{j}}{\left(q, a b c d q^{k} ; q\right)_{j}} q^{k+j} .
\end{aligned}
$$

Proof Letting

$$
f_{L}=f_{L}\left(h_{0}, \ldots, h_{t}, g_{1}, \ldots, g_{t}, b, f\right)=\frac{1}{(b x ; q)_{\infty}} s+1 \Phi_{s}\left(\begin{array}{c}
h_{0}, h_{1}, \ldots, h_{t} \\
g_{1}, \ldots, g_{t}
\end{array} ;, f x\right)
$$

and

$$
\begin{aligned}
f_{R} & =f_{R}\left(h_{0}, \ldots, h_{t}, g_{1}, \ldots, g_{t}, b, f\right) \\
& =\frac{\left(a b c d q^{k} ; q\right)_{\infty}}{(b c, b d ; q)_{\infty}} \sum_{l=0}^{\infty} \frac{\left(h_{0}, h_{1}, \ldots, h_{t} ; q\right)_{l}}{\left(q, g_{1}, \ldots, g_{t} ; q\right)_{l}}\left(\frac{f}{b}\right)^{l}{ }_{3} \Phi_{2}\left(\begin{array}{c}
q^{-n}, b c, b d \\
0, a b c d q^{k}
\end{array} q, q\right),
\end{aligned}
$$

we can easily verify both of the above identities satisfy (6), so we have

$$
\begin{aligned}
f_{L} & =F\left(h_{0}, \ldots, h_{t} ; g_{1}, \ldots, g_{t}, f D_{q, b}\right)\left\{f_{L}\left(h_{0}, \ldots, h_{t}, g_{1}, \ldots, g_{t}, b, 0\right)\right\} \\
& =F\left(h_{0}, \ldots, h_{t} ; g_{1}, \ldots, g_{t}, f D_{q, b}\right)\left\{\frac{1}{(b x ; q)_{\infty}}\right\}
\end{aligned}
$$

and

$$
\begin{aligned}
f_{R} & =F\left(h_{0}, \ldots, h_{t} ; g_{1}, \ldots, g_{t}, f D_{q, b}\right)\left\{f_{R}\left(h_{0}, \ldots, h_{t}, g_{1}, \ldots, g_{t}, b, 0\right)\right\} \\
& =F\left(h_{0}, \ldots, h_{t} ; g_{1}, \ldots, g_{t}, f D_{q, b}\right)\left\{\frac{\left(a b c d q^{k} ; q\right)_{\infty}}{(b c, b d ; q)_{\infty}}\right\} .
\end{aligned}
$$

From (41), we conclude that

$$
\begin{aligned}
& \int_{c}^{d} \frac{(q x / c, q x / d ; q)_{\infty}}{(a x, b x ; q)_{\infty}}{ }_{s+1} \Phi_{s}\left(\begin{array}{c}
a_{0}, a_{1}, \ldots, a_{s} \\
b_{1}, \ldots, b_{s}
\end{array} q, e x\right) d_{q} x \\
& \quad=\frac{d(1-q)(q, d q / c, c / d ; q)_{\infty}}{(a c, a d ; q)_{\infty}} \sum_{n=0}^{\infty} \sum_{k=0}^{n} W_{n}\left(\frac{e}{a}\right) \frac{\left(q^{-n}, a c, a d ; q\right)_{k} q^{k}}{(q, a b c d ; q)_{k}} \frac{\left(a b c d q^{k} ; q\right)_{\infty}}{(b c, b d ; q)_{\infty}} .
\end{aligned}
$$

By (51) and (52), we complete the proof. 
Interchanging $a$ and $b$ in (48), similar to (47), we find the following.

Corollary 2.9 We have

$$
\begin{aligned}
& a^{n-l} \sum_{k=0}^{n} \frac{\left(q^{-n}, a c, a d ; q\right)_{k} q^{k}}{(q, a b c d ; q)_{k}}{ }_{3} \Phi_{2}\left(\begin{array}{l}
q^{-l}, b c, b d \\
0, a b c d q^{k}
\end{array} ;, q\right) \\
& \quad=b^{n-l} \sum_{k=0}^{n} \frac{\left(q^{-n}, b c, b d ; q\right)_{k} q^{k}}{(q, a b c d ; q)_{k}}{ }_{3} \Phi_{2}\left(\begin{array}{c}
q^{-l}, a c, a d \\
0, a b c d q^{k}
\end{array} q, q\right) .
\end{aligned}
$$

Setting $a=d=q, b=c=-q$ in (54), then letting $q \rightarrow 1$, we have the following.

Corollary 2.10 If $n-l=1(\bmod 2)$, then

$$
\sum_{k=0}^{n} \sum_{j=0}^{l}\left(\begin{array}{l}
n \\
k
\end{array}\right)\left(\begin{array}{l}
l \\
j
\end{array}\right) \frac{3 \cdot 2^{k+1}}{(k+2)(k+3)} \frac{2^{j}(j+1) !}{(4+k)_{j}}(-1)^{k+j}=0,
$$

where $(a)_{0}=1,(a)_{j}=a(a+1) \cdots(a+j-1)$.

\section{Generalizations of Askey-Wilson integral}

In [12], we had derived a new of $q$-contour integral formula from the following elegant Askey-Wilson integral formula (cf. [6], Theorem 2.1):

$$
\frac{1}{2 \pi i} \int_{C} \frac{\left(z^{2}, z^{-2} ; q\right)_{\infty}}{(a z, a / z, b z, b / z, c z, c / z, d z, d / z ; q)_{\infty}} \frac{d z}{z}=\frac{2(a b c d ; q)_{\infty}}{(q, a b, a c, a d, b c, b d, c d ; q)_{\infty}},
$$

where the contour $C$ is a deformation of unit circle so that the poles of $1 /(a z, b z, c z, d z ; q)_{\infty}$ lie outside the contour and the origin and poles of $1 /(a / z, b / z, c / z, d / z ; q)_{\infty}$ lie inside the contour. In this section, we get the following generalizations of the above equation.

Theorem 3.1 If $a_{0}=q^{-N}, a_{i}, b_{i} \in \mathbb{C}, i=1,2, \ldots, s, N \in \mathbb{N}$, then

$$
\begin{aligned}
& \frac{1}{2 \pi i} \int_{C} \frac{\left(z^{2}, z^{-2} ; q\right)_{\infty}}{(a z, a / z, b z, b / z, c z, c / z, d z, d / z ; q)_{\infty}} \\
& \quad \times \sum_{n=0}^{\infty} W_{n}\left(\frac{e}{a}\right)^{n}{ }_{3} \Phi_{2}\left(\begin{array}{c}
q^{-n}, a z, a / z \\
0, a c
\end{array} q, q\right) \frac{d z}{z} \\
& =\frac{2(a b c d ; q)_{\infty}}{(q, a b, a c, a d, b c, b d, c d ; q)_{\infty}} \sum_{n=0}^{\infty} W_{n}\left(\frac{e}{a}\right)^{n}{ }_{3} \Phi_{2}\left(\begin{array}{c}
q^{-n}, a b, a d \\
0, a b c d
\end{array} ;, q\right) .
\end{aligned}
$$

Proof We rewrite (57) as follows:

$$
\begin{aligned}
& \frac{1}{2 \pi i} \int_{C} \frac{\left(z^{2}, z^{-2} ; q\right)_{\infty}}{(b z, b / z, c z, c / z, d z, d / z ; q)_{\infty}} \frac{(a c ; q)_{\infty}}{(a z, a / z ; q)_{\infty}} \\
& \quad \times \sum_{n=0}^{\infty} W_{n}\left(\frac{e}{a}\right)^{n}{ }_{3} \Phi_{2}\left(\begin{array}{r}
q^{-n}, a z, a / z \\
0, a c
\end{array} q, q\right) \frac{d z}{z} \\
& =\frac{2}{(q, b c, b d, c d ; q)_{\infty}} \frac{(a b c d ; q)_{\infty}}{(a b, a d ; q)_{\infty}} \sum_{n=0}^{\infty} W_{n}\left(\frac{e}{a}\right)^{n}{ }_{3} \Phi_{2}\left(\begin{array}{r}
q^{-n}, a b, a d \\
0, a b c d
\end{array} ;, q\right) .
\end{aligned}
$$


We use $f_{L}=f_{L}\left(a_{0}, \ldots, a_{s}, b_{1}, \ldots, b_{s}, a, e\right)$ and $f_{R}=f_{R}\left(a_{0}, \ldots, a_{s}, b_{1}, \ldots, b_{s}, a, e\right)$ to denote the left-hand and the right-hand side of (58), respectively. By the same method as in Theorem 1.4 , we can verify they both satisfy (6). Letting $F=F\left(a_{0}, \ldots, a_{s} ; b_{1}, \ldots, b_{s} ; e D_{q, a}\right)$, we have

$$
\begin{aligned}
f_{R} & =F\left\{f_{R}\left(a_{0}, \ldots, a_{s}, b_{1}, \ldots, b_{s}, a, 0\right)\right\}=F\left\{\frac{2}{(q, b c, b d, c d ; q)_{\infty}} \frac{(a b c d ; q)_{\infty}}{(a b, a d ; q)_{\infty}}\right\} \\
& =\frac{1}{2 \pi i} \int_{C} \frac{\left(z^{2}, z^{-2} ; q\right)_{\infty}}{(b z, b / z, c z, c / z, d z, d / z ; q)_{\infty}} F\left\{\frac{(a c ; q)_{\infty}}{(a z, a / z ; q)_{\infty}}\right\} \frac{d z}{z} .
\end{aligned}
$$

Applying (16), the above identity is equal to the left side of (58). This completes the proof.

Employing the above theorem, using $q$-operator $F=F\left(h_{0}, \ldots, h_{t} ; g_{1}, \ldots, g_{t} ; f D_{q, b}\right)$, similar to the above proof, we conclude the following.

Theorem 3.2 If $a_{0}=q^{-N}, h_{0}=q^{-G}, a_{i}, b_{i}, h_{j}, g_{j} \in \mathbb{C}, i=1, \ldots, s, j=1, \ldots, t, G, N \in \mathbb{N}$, then

$$
\begin{aligned}
& \frac{1}{2 \pi i} \int_{C} \frac{\left(z^{2}, z^{-2} ; q\right)_{\infty}}{(a z, a / z, b z, b / z, c z, c / z, d z, d / z ; q)_{\infty}} \sum_{n=0}^{\infty} W_{n}\left(\frac{e}{a}\right)^{n}{ }_{3} \Phi_{2}\left(\begin{array}{c}
q^{-n}, a z, a / z \\
0, a c
\end{array} ;, q\right) \\
& \quad \times \sum_{m=0}^{\infty} U_{m}\left(\frac{f}{b}\right)^{n}{ }_{3} \Phi_{2}\left(\begin{array}{c}
q^{-m}, b z, b / z \\
0, b c
\end{array} q, q\right) \frac{d z}{z} \\
& =\frac{2(a b c d ; q)_{\infty}}{(q, a b, a c, a d, b c, b d, c d ; q)_{\infty}} \sum_{n=0}^{\infty} \sum_{m=0}^{\infty} \sum_{k=0}^{n} \sum_{l=0}^{m} W_{n} U_{m}\left(\frac{e}{a}\right)^{n}\left(\frac{f}{b}\right)^{m} \\
& \quad \times \frac{\left(q^{-n}, a b, a d ; q\right)_{k}}{(q, a b c d ; q)_{k}} \frac{\left(q^{-m}, a b q^{k}, a d ; q\right)_{l}}{\left(q, a b c d q^{k} ; q\right)_{l}} q^{k+l} .
\end{aligned}
$$

\section{Some properties of $q$-polynomials $\boldsymbol{H}_{\boldsymbol{n}}$}

For $a_{0}, a_{1}, \ldots, a_{s}, b_{1}, \ldots, b_{s}, b, c \in \mathbb{C}, s \in \mathbb{N}$, we define

$$
\begin{aligned}
H_{n} & =H_{n}\left(a_{0}, a_{1}, \ldots, a_{s} ; b_{1}, \ldots, b_{s} ; b, c\right)=\sum_{k=0}^{n}\left[\begin{array}{l}
n \\
k
\end{array}\right] \frac{\left(a_{0}, a_{1}, \ldots, a_{s} ; q\right)_{k}}{\left(b_{1}, \ldots, b_{s} ; q\right)_{k}} c^{k} b^{n-k} \\
& =b^{n}{ }_{s+2} \Phi_{s}\left(\begin{array}{c}
q^{-n}, a_{0}, a_{1}, a_{2}, \ldots, a_{s} \\
b_{1}, b_{2}, \ldots, b_{s}
\end{array} q, q^{n} c / b\right) .
\end{aligned}
$$

We can get some famous polynomials from $H_{n}, e . g$, letting $b=1, a_{i}=b_{i}=0, i=1,2, \ldots, s$, the polynomials $H_{n}$ reduce to the classical Al-Salam-Carlitz polynomials (cf. [1], Eq. (1.11)),

$$
\Phi_{n}^{\left(a_{0}\right)}(c)=\Phi_{n}^{\left(a_{0}\right)}(c, q)=\sum_{k=0}^{n}\left[\begin{array}{l}
n \\
k
\end{array}\right]\left(a_{0} ; q\right)_{k} c^{k}
$$

Setting $b=1, c=q / a_{0} q^{n}$ in (61), then letting $a_{0} \rightarrow \infty$, we have

$$
\lim _{a_{0} \rightarrow \infty} H_{n}\left(a_{0}, a_{1}, \ldots, a_{s} ; b_{1}, \ldots, b_{s} ; 1, q / a_{0} q^{n}\right)={ }_{s+1} \Phi_{s}\left(\begin{array}{c}
q^{-n}, a_{1}, a_{2}, \ldots, a_{s} \\
b_{1}, b_{2}, \ldots, b_{s}
\end{array} ;, q\right) .
$$


Taking $a_{1}=a b c d q^{n-1}, a_{2}=a e^{i \theta}, a_{3}=a e^{-i \theta}, b_{1}=a b, b_{2}=a c, b_{3}=a d, a_{i}=b_{i}=0, i=4, \ldots, s$ in (63), we have the Askey-Wilson polynomials ([6], Eq. (1.15))

$$
\frac{a^{n} p_{n}(x ; a, b, c, d \mid q)}{(a b, a c, a d ; q)_{n}}={ }_{4} \Phi_{3}\left(\begin{array}{r}
q^{-n}, a b c d q^{n-1}, a e^{i \theta}, a e^{-i \theta} \\
a b, a c, a d
\end{array} ; q, q\right) .
$$

Putting $a_{1}=a b q^{n+1}, a_{2}=q^{-x}, a_{3}=c q^{x-N}, b_{1}=a q, b_{2}=q^{-N}, b_{3}=b c q, a_{i}=b_{i}=0, i=4, \ldots, s$ in (63), we get the $q$-Racah polynomials ([15], Eq. (7.2.17)),

$$
W_{n}(x ; a, b, c, N \mid q)={ }_{4} \Phi_{3}\left(\begin{array}{r}
q^{-n}, a b q^{n+1}, q^{-x}, c q^{x-N} \\
a q, q^{-N}, b c q
\end{array} ; q, q\right)
$$

In this section, we will give some properties of $q$-polynomials $H_{n}$ by $q$-difference equation. We now show the $H_{n}$ satisfies the following $q$-difference equation.

Theorem 4.1 If $a_{0}, a_{1}, \ldots, a_{s}, b_{1}, \ldots, b_{s}, b, c \in \mathbb{C}, s \in \mathbb{N}$, then

$$
\begin{aligned}
& b \sum_{j=0}^{s+1} \frac{(-1)^{j} B_{j}}{q^{j}} H_{n}\left(a_{0}, \ldots, b_{1}, \ldots, b_{s}, b, c q^{j}\right)-c \sum_{j=0}^{s+1}(-1)^{j} A_{j}\left[H_{n}\left(a_{0}, \ldots, b_{1}, \ldots, b_{s}, b, c q^{j}\right)\right. \\
& \left.\quad-H_{n}\left(a_{0}, \ldots, a_{s}, b_{1}, \ldots, b_{s}, b q, c q^{j}\right)\right]=0,
\end{aligned}
$$

where $A_{j}, B_{j}$ are defined as (7).

Proof Letting $W_{n}$ defined as (17), and denoting $W_{n}=W_{n}^{\prime} /(q ; q)_{n}$, we have

$$
\begin{aligned}
& b \sum_{j=0}^{s+1} \frac{(-1)^{j} B_{j}}{q^{j}} H_{n}\left(a_{0}, \ldots, b_{1}, \ldots, b_{s}, b, c q^{j}\right) \\
& \quad=b \sum_{k=0}^{n} \frac{b^{n-k} c^{k}(q ; q)_{n}}{(q ; q)_{n-k}} W_{k}\left[1-B_{1} q^{k-1}+\cdots+(-1)^{s+1} B_{s+1} q^{(s+1)(k-1)}\right] \\
& \quad=c \sum_{k=0}^{n} \frac{b^{n-(k-1)} c^{k-1}(q ; q)_{n-1}\left(1-q^{n}\right)}{(q ; q)_{n-k}} W_{k-1}\left(1-a_{0} q^{k-1}\right) \cdots\left(1-a_{s} q^{k-1}\right) \\
& \quad=c\left(1-q^{n}\right) \sum_{k=0}^{n}\left[\begin{array}{c}
n-1 \\
k-1
\end{array}\right] b^{n-(k-1)} c^{k-1} W_{k-1}^{\prime}\left(1-a_{0} q^{k-1}\right) \cdots\left(1-a_{s} q^{k-1}\right) .
\end{aligned}
$$

Replacing $k-1$ by $k$, we find that the above equation is equal to

$$
c\left(1-q^{n}\right) \sum_{k=0}^{n}\left[\begin{array}{c}
n-1 \\
k
\end{array}\right] b^{n-k} c^{k} W_{k}^{\prime}\left(1-a_{0} q^{k}\right) \cdots\left(1-a_{s} q^{k}\right) .
$$

On the other hand

$$
\begin{aligned}
& c \sum_{j=0}^{s+1}(-1)^{j} A_{j}\left[H_{n}\left(a_{0}, \ldots, b_{1}, \ldots, b_{s}, b, c q^{j}\right)-H_{n}\left(a_{0}, \ldots, a_{s}, b_{1}, \ldots, b_{s}, b q, c q^{j}\right)\right] \\
& \quad=c \sum_{k=0}^{n}\left[\begin{array}{l}
n \\
k
\end{array}\right] b^{n-k}\left(1-q^{n-k}\right) c^{k} W_{k}^{\prime}\left(1-a_{0} q^{k}\right) \cdots\left(1-a_{s} q^{k}\right)
\end{aligned}
$$




$$
\begin{aligned}
= & c \sum_{k=0}^{n}\left(\left[\begin{array}{c}
n-1 \\
k
\end{array}\right]+\left[\begin{array}{c}
n-1 \\
k-1
\end{array}\right] q^{n-k}\right) b^{n-k} c^{k} W_{k}^{\prime}\left(1-a_{0} q^{k}\right) \cdots\left(1-a_{s} q^{k}\right) \\
& -c \sum_{k=0}^{n}\left(\left[\begin{array}{c}
n-1 \\
k
\end{array}\right] q^{k}+\left[\begin{array}{c}
n-1 \\
k-1
\end{array}\right]\right)(b q)^{n-k} c^{k} W_{k}^{\prime}\left(1-a_{0} q^{k}\right) \cdots\left(1-a_{s} q^{k}\right) \\
= & c\left(1-q^{n}\right) \sum_{k=0}^{n}\left[\begin{array}{c}
n-1 \\
k
\end{array}\right] b^{n-k} c^{k} W_{k}^{\prime}\left(1-a_{0} q^{k}\right) \cdots\left(1-a_{s} q^{k}\right) .
\end{aligned}
$$

This completes the proof.

For $H_{n}$ satisfies (6), applying (8), we find the following.

Corollary 4.2 If $H_{n}\left(a_{0}, a_{1}, \ldots, a_{s} ; b_{1}, \ldots, b_{s} ; b, c\right)$ is defined as (61), then

$$
H_{n}\left(a_{0}, a_{1}, \ldots, a_{s} ; b_{1}, \ldots, b_{s} ; b, c\right)=F\left(a_{0}, a_{1}, \ldots, a_{s} ; b_{1}, \ldots, b_{s} ; c D_{q, b}\right)\left\{b^{n}\right\}
$$

Combining the above equation and (23), we obtain the following generating functions for $H_{n}$.

Theorem 4.3 If $\max \{|b u|,|c u|\}<1$, then

$$
\sum_{n=0}^{\infty} H_{n} \frac{u^{n}}{(q ; q)_{n}}=\frac{1}{(b u ; q)_{\infty}} \sum_{n=0}^{\infty} W_{n}(c u)^{n}
$$

Setting $b=1, a_{i}=b_{i}=0, i=1,2, \ldots, s$ in (71), we conclude the following.

Corollary 4.4 ([1], Eq. (1.13)) If $\max \{|u|,|c u|\}<1$, then

$$
\sum_{n=0}^{\infty} \Phi_{n}^{(a)}(c) \frac{u^{n}}{(q ; q)_{n}}=\frac{\left(a_{0} c u ; q\right)_{\infty}}{(u, c u ; q)_{\infty}}
$$

Theorem 4.5 If $a_{0}=q^{-N}, h_{0}=q^{-G}, G, N \in \mathbb{N}, \max \{|b e v|,|c e v|,|b f v|\}<1$, then

$$
\begin{gathered}
\sum_{n=0}^{\infty} H_{n}\left(a_{0}, a_{1}, \ldots, a_{s} ; b_{1}, \ldots, b_{s} ; b, c\right) H_{n}\left(h_{0}, h_{1}, \ldots, h_{t} ; g_{1}, \ldots, g_{t} ; e, f\right) \frac{v^{n}}{(q ; q)_{n}} \\
\quad=\frac{1}{(b e v ; q)_{\infty}} \sum_{n=0}^{\infty} \sum_{m=0}^{\infty} \sum_{k=0}^{m}\left[\begin{array}{l}
m \\
k
\end{array}\right]\left[\begin{array}{l}
n \\
k
\end{array}\right] W_{m} U_{n} \frac{(q, b e v ; q)_{k}}{(b e v)^{k}}(b f v)^{n}(c e v)^{m}
\end{gathered}
$$

To prove the above theorem, we need the following lemma.

Lemma 4.6 If $a_{0}=q^{-N}, v, u, a_{i}, b_{i} \in \mathbb{C}, i=1,2, \ldots, s, s, N \in \mathbb{N}$, then

$$
\begin{aligned}
& F\left(a_{0}, a_{1}, \ldots, a_{s} ; b_{1}, \ldots, b_{s} ; c D_{q, b}\right)\left\{\frac{(b v)^{n}}{(b u ; q)_{\infty}}\right\} \\
& =\frac{(b v)^{n}}{(b u ; q)_{\infty}} \sum_{m=0}^{\infty} \sum_{k=0}^{m}\left[\begin{array}{l}
m \\
k
\end{array}\right]\left[\begin{array}{l}
n \\
k
\end{array}\right] W_{m}(c u)^{m} \frac{(q, b u ; q)_{k}}{(b u)^{k}} .
\end{aligned}
$$


Proof Letting $f\left(a_{0}, a_{1}, \ldots, a_{s} ; b_{1}, \ldots, b_{s} ; b, c\right)$ denoting the right-hand side of (74), similar to the proof of Theorem 1.4, we see that the functions $f\left(a_{0}, a_{1}, \ldots, a_{s} ; b_{1}, \ldots, b_{s} ; b, c\right)$ satisfies (6). Applying (8), we complete the proof.

Proof of Theorem 4.5 The left-hand side of (73) is equal to

$$
\begin{aligned}
& \sum_{n=0}^{\infty} F\left(a_{0}, a_{1}, \ldots, a_{s} ; b_{1}, \ldots, b_{s} ; c D_{q, b}\right)\left\{b^{n}\right\} H_{n}\left(a_{0}, a_{1}, \ldots, a_{s} ; b_{1}, \ldots, b_{s} ; e, f\right) \frac{v^{n}}{(q ; q)_{n}} \\
& \quad=F\left(a_{0}, a_{1}, \ldots, a_{s} ; b_{1}, \ldots, b_{s} ; c D_{q, b}\right)\left\{\sum_{n=0}^{\infty} H_{n}\left(a_{0}, a_{1}, \ldots, a_{s} ; b_{1}, \ldots, b_{s} ; e, f\right) \frac{(b v)^{n}}{(q ; q)_{n}}\right\} \\
& \quad=F\left(a_{0}, a_{1}, \ldots, a_{s} ; b_{1}, \ldots, b_{s} ; c D_{q, b}\right)\left\{\frac{1}{(b e v ; q)_{\infty}} \sum_{n=0}^{\infty} W_{n}(b f v)^{n}\right\}
\end{aligned}
$$

Using Lemma 4.6, we complete the proof.

Theorem 4.7 If $a_{0}=q^{-N}, a_{i}, b_{i} \in \mathbb{C}, i=1,2, \ldots, s, s, N \in \mathbb{N}, \max \{|b u|,|b w|,|b v|\}<1$, then

$$
\begin{aligned}
& \sum_{m, n, k=0}^{\infty} H_{m+n+k} \frac{u^{m} v^{n} w^{k}(-1)^{k} q^{\left(\frac{k}{2}\right)}}{(q ; q)_{m}(q ; q)_{n}(q ; q)_{k}} \\
& \quad=\frac{(b w ; q)_{\infty}}{(b u, b v ; q)_{\infty}} \sum_{n=0}^{\infty} W_{n}\left(\frac{c}{b}\right)^{n}{ }_{3} \Phi_{2}\left(\begin{array}{r}
q^{-n}, b u, b v \\
0, b w
\end{array} ;, q\right) .
\end{aligned}
$$

Proof The left-hand side of (76) is equal to

$$
\begin{aligned}
& F\left(a_{0}, a_{1}, \ldots, a_{s} ; b_{1}, \ldots, b_{s} ; c D_{q, b}\right)\left\{\sum_{m, n, k=0}^{\infty} \frac{(b u)^{m}(b v)^{n}(b w)^{k}(-1)^{k} q^{\left(\frac{k}{2}\right)}}{(q ; q)_{m}(q ; q)_{n}(q ; q)_{k}}\right\} \\
& \quad=F\left(a_{0}, a_{1}, \ldots, a_{s} ; b_{1}, \ldots, b_{s} ; c D_{q, b}\right)\left\{\frac{(b w ; q)_{\infty}}{(b u, b v ; q)_{\infty}}\right\} .
\end{aligned}
$$

By Theorem 1.4, the proof is complete.

Letting $w=0$ in (76), we have the following.

Corollary 4.8 If $a_{0}=q^{-N}, a_{i}, b_{i} \in \mathbb{C}, i=1,2, \ldots, s, s, N \in \mathbb{N}, \max \{|b u|,|b v|\}<1$, then

$$
\sum_{m, n=0}^{\infty} H_{m+n} \frac{u^{m} v^{n}}{(q ; q)_{m}(q ; q)_{n}}=\frac{1}{(b u, b v ; q)_{\infty}} \sum_{n=0}^{\infty} W_{n}\left(\frac{c}{b}\right)^{n}{ }_{3} \Phi_{2}\left(\begin{array}{r}
q^{-n}, b u, b v \\
0,0
\end{array} ; q, q\right) .
$$

Setting $v=0$ in (76), then applying (24), we find the following.

Corollary 4.9 If $\max \{|b u|,|b w|,|c u|\}<1$, then

$$
\sum_{m, k=0}^{\infty} H_{m+k} \frac{u^{m} w^{k}(-1)^{k} q^{\left(\begin{array}{c}
k \\
2
\end{array}\right)}}{(q ; q)_{m}(q ; q)_{k}}=\frac{(b w ; q)_{\infty}}{(b u ; q)_{\infty}} \sum_{n=0}^{\infty} \frac{\left(a_{0}, a_{1}, \ldots, a_{s}, w / u ; q\right)_{n}}{\left(q, b_{1}, \ldots, b_{s}, b w ; q\right)_{n}}(c u)^{n}
$$




\section{Some special cases}

In this section, we briefly consider some consequences and special cases of the results derived in Section 2. If we take $e=q / d, a_{i}=b_{i}=0, i=2,3, \ldots, s$ in (27), applying (24), we obtain the following.

Proposition 5.1 If $a_{0}=q^{-N}, a_{1}, b_{1} \in \mathbb{C}, N \in \mathbb{N}$, then

$$
\begin{gathered}
\int_{c}^{d} \frac{(q x / c, q x / d ; q)_{\infty}}{(a x, b x ; q)_{\infty}} \sum_{n=0}^{N} \frac{\left(q^{-N}, a_{1} ; q\right)_{n}}{\left(q, b_{1} ; q\right)_{n}}\left(\frac{q}{a d}\right)^{n}{ }_{3} \Phi_{2}\left(\begin{array}{r}
q^{-n}, a x, a b c d \\
0, a c
\end{array} q, q\right) d_{q} x \\
=\frac{d(1-q)(q, d q / c, c / d, a b c d ; q)_{\infty}\left(b_{1} / a_{1} ; q\right)_{N} a_{1}{ }^{N}}{(a c, a d, b c, b d ; q)_{\infty}\left(b_{1} ; q\right)_{N}}
\end{gathered}
$$

If we take $e=b_{1} / a_{0} c, a_{1}=a c, a_{i}=b_{i}=0, i=2,3, \ldots, s$ in (38), applying the $q$-Gauss summation ([15], p.354, Eq. (II.8))

$$
{ }_{2} \Phi_{1}\left(\begin{array}{c}
a, b \\
c
\end{array} ;, c / a b\right)=\frac{(c / a, c / b ; q)_{\infty}}{(c, c / a b ; q)_{\infty}}
$$

then replacing $\left(b_{1}, a_{0}\right)$ by $\left(b_{1} a_{0} c, a_{0} / b_{1}\right)$, respectively, we get

$$
\begin{aligned}
& \int_{c}^{d} \frac{\left(q x / c, q x / d, a_{0} x ; q\right)_{\infty}}{\left(a x, b x, b_{1} x ; q\right)_{\infty}} d_{q} x \\
& \quad=\frac{d(1-q)\left(q, d q / c, c / d, a b c d, a_{0} c ; q\right)_{\infty}}{\left(a c, a d, b c, b d, b_{1} c ; q\right)_{\infty}} \Phi_{2}\left(\begin{array}{c}
a c, a_{0} / b_{1}, b c \\
a_{0} c, a b c d
\end{array} ;, d b_{1}\right) .
\end{aligned}
$$

For ${ }_{3} \Phi_{2}$ series, using Hall's transformation ([15], p.359, Eq. (III.10))

$$
{ }_{3} \Phi_{2}\left(\begin{array}{c}
a, b, c \\
d, e
\end{array} ;, d e / a b c\right)=\frac{(b, d e / a b, d e / b c ; q)_{\infty}}{(d, e, d e / a b c ; q)_{\infty}}{ }_{3} \Phi_{2}\left(\begin{array}{c}
d / b, e / b, d e / a b c \\
d e / a b, d e / b c
\end{array} ;, b\right),
$$

we find the following.

Proposition 5.2 ([20], Theorem 9) We have

$$
\begin{gathered}
\int_{c}^{d} \frac{\left(q x / c, q x / d, a_{0} x ; q\right)_{\infty}}{\left(a x, b x, b_{1} x ; q\right)_{\infty}} d_{q} x \\
=\frac{d(1-q)\left(q, d q / c, c / d, a_{0} / b_{1}, a c d b_{1}, b c d b_{1} ; q\right)_{\infty}}{\left(a c, a d, b c, b d, b_{1} c, b_{1} d ; q\right)_{\infty}} \\
\quad \times{ }_{3} \Phi_{2}\left(\begin{array}{c}
b_{1} c, b_{1} d, a b c d b_{1} / a_{0} \\
a c d b_{1}, b c d b_{1}
\end{array} q, a_{0} / b_{1}\right) .
\end{gathered}
$$

Setting $a_{0}=a b c d b_{1}$ in the above identity, we obtain the following.

Proposition 5.3 ([20], Theorem 8) We have

$$
\int_{c}^{d} \frac{\left(q x / c, q x / d, a b c d b_{1} x ; q\right)_{\infty}}{\left(a x, b x, b_{1} x ; q\right)_{\infty}} d_{q} x=\frac{d(1-q)\left(q, d q / c, c / d, a b c d, a c d b_{1}, b c d b_{1} ; q\right)_{\infty}}{\left(a c, a d, b c, b d, b_{1} c, b_{1} d ; q\right)_{\infty}} .
$$


Noting

$$
\int_{c}^{d} f(x) d_{q} x=d(1-q) \sum_{m=0}^{\infty} f\left(d q^{m}\right) q^{m}-c(1-q) \sum_{m=0}^{\infty} f\left(c q^{m}\right) q^{m}
$$

then letting $a=q, b=-q, c=-1, d=1$ in (41), we get the following.

Proposition 5.4 If $G \in \mathbb{N}$, we get

$$
\begin{aligned}
& \sum_{k=0}^{G}\left[\begin{array}{c}
G \\
k
\end{array}\right] e^{k} q^{\left(\begin{array}{c}
k \\
2
\end{array}\right)-G k} \frac{\left(a_{1}, \ldots, a_{s} ; q\right)_{k}}{\left(b_{1}, \ldots, b_{s} ; q\right)_{k}} \frac{(1-q)\left(1+(-1)^{k}\right)}{1-q^{k+1}} \\
& \quad=2 \sum_{n=0}^{G} \sum_{k=0}^{n}\left[\begin{array}{l}
G \\
n
\end{array}\right]\left[\begin{array}{l}
n \\
k
\end{array}\right]\left(-\frac{e}{q}\right)^{n} q^{\left(\begin{array}{c}
k+1 \\
2
\end{array}\right)+\left(\begin{array}{c}
n \\
2
\end{array}\right)-G n-n k} \frac{\left(a_{1}, \ldots, a_{s} ; q\right)_{n}}{\left(b_{1}, \ldots, b_{s} ; q\right)_{n}}(-1)^{k} \frac{\left(q^{2} ; q^{2}\right)_{k}}{\left(q^{2} ; q\right)_{k}} .
\end{aligned}
$$

Taking $e=-q, a_{i}=q, b_{i}=q^{2}, i=1,2, \ldots, 2$ in (87), then letting $q \rightarrow 1$ yields the following.

Corollary 5.5 If $s, G \in \mathbb{N}$, then

$$
\sum_{n=0}^{[G / 2]}\left(\begin{array}{c}
G \\
2 n
\end{array}\right) \frac{1}{(2 n+1)^{s+1}}=\sum_{n=0}^{G} \sum_{k=0}^{n}\left(\begin{array}{l}
G \\
n
\end{array}\right)\left(\begin{array}{l}
n \\
k
\end{array}\right) \frac{1}{(n+1)^{s}} \frac{(-1)^{k} 2^{k}}{k+1}
$$

If let $e=q, a_{i}=q, b_{i}=q^{2}, i=1,2, \ldots, 2$ in (87), and setting $q \rightarrow 1$, we have the following.

Corollary 5.6 If $s, G \in \mathbb{N}$, then

$$
\sum_{n=0}^{[G / 2]}\left(\begin{array}{c}
G \\
2 n
\end{array}\right) \frac{1}{(2 n+1)^{s+1}}=\sum_{n=0}^{G} \sum_{k=0}^{n}\left(\begin{array}{l}
G \\
n
\end{array}\right)\left(\begin{array}{l}
n \\
k
\end{array}\right) \frac{(-1)^{n}}{(n+1)^{s}} \frac{(-1)^{k} 2^{k}}{k+1}
$$

Combining with the above two identities, we obtain the following.

Corollary 5.7 If $s, G \in \mathbb{N}$, then

$$
\sum_{m=0}^{[G-1 / 2]} \sum_{k=0}^{2 m+1}\left(\begin{array}{c}
G \\
2 m+1
\end{array}\right)\left(\begin{array}{c}
2 m+1 \\
k
\end{array}\right) \frac{1}{(2 m+2)^{s}} \frac{(-1)^{k} 2^{k}}{k+1}=0
$$

Taking $e=-q, a_{i}=q^{2}, b_{i}=q, i=1,2, \ldots, 2$ in (87), then letting $q \rightarrow 1$ yields the following.

Corollary 5.8 If $s, G \in \mathbb{N}$, then

$$
\sum_{n=0}^{[G / 2]}\left(\begin{array}{c}
G \\
2 n
\end{array}\right)(2 n+1)^{s-1}=\sum_{n=0}^{G} \sum_{k=0}^{n}\left(\begin{array}{l}
G \\
n
\end{array}\right)\left(\begin{array}{l}
n \\
k
\end{array}\right)(n+1)^{s} \frac{(-1)^{k} 2^{k}}{k+1}
$$

Setting $e=q, a_{i}=q^{2}, b_{i}=q, i=1,2, \ldots, 2$ in (87), then letting $q \rightarrow 1$ yields the following.

Corollary 5.9 If $s, G \in \mathbb{N}$, then

$$
\sum_{n=0}^{[G / 2]}\left(\begin{array}{c}
G \\
2 n
\end{array}\right)(2 n+1)^{s-1}=\sum_{n=0}^{G} \sum_{k=0}^{n}\left(\begin{array}{l}
G \\
n
\end{array}\right)\left(\begin{array}{l}
n \\
k
\end{array}\right)(-1)^{n}(n+1)^{s} \frac{(-1)^{k} 2^{k}}{k+1}
$$


Combining with the above two identities, we obtain the following.

Corollary 5.10 If $s, G \in \mathbb{N}$, then

$$
\sum_{m=0}^{[G-1 / 2]} \sum_{k=0}^{2 m+1}\left(\begin{array}{c}
G \\
2 m+1
\end{array}\right)\left(\begin{array}{c}
2 m+1 \\
k
\end{array}\right)(2 m+2)^{s} \frac{(-1)^{k} 2^{k}}{k+1}=0
$$

Remark 5.11 The symbol $[x]$ denotes the largest integer $\leq x$.

\section{Competing interests}

The author declares that he has no competing interests.

\section{Author's contributions}

The author read and approved the final manuscript.

\section{Acknowledgements}

The author would like to thank the editors and the anonymous referees for their careful reading of the paper. The author is supported by National Natural Science Foundation of China (No. 11471138) and National Natural Science Foundation of China (No. 11371163). The author is also supported by Jiangsu Overseas Research and Training Program for University Prominent Young and Middle-Aged Teachers and Presidents, Universities Natural Science Foundation of Jiangsu (No. 14KJB110002) and SRF for ROCS, SEM.

Received: 14 July 2014 Accepted: 30 September 2014 Published: 14 Oct 2014

\section{References}

1. Al-Salam, WA, Carlitz, L: Some orthogonal q-polynomials. Math. Nachr. 30, 47-61 (1965)

2. Andrews, GE: $q$-Series: Their Development and Application in Analysis, Number Theory, Combinatorics, Physics, and Computer Algebra. Conference Series in Mathematics, vol. 66. Am. Math. Soc., Providence (1986)

3. Andrews, GE, Askey, R: Another q-extension of the beta function. Proc. Am. Math. Soc. 81, 97-100 (1981)

4. Askey, R: The q-gamma and q-beta function. Appl. Anal. 8, 125-141 (1978)

5. Askey, R: A q-extension of Cauchy's form of the beta integral. Q. J. Math. 32(3), 255-266 (1981)

6. Askey, R, Wilson, J: Some basic hypergeometric polynomials that generalize Jacobi polynomials. Mem. Am. Math. Soc. 54,319 (1985)

7. Bowman, D: q-Differential operators, orthogonal polynomials, and symmetric expansions. Mem. Am. Math. Soc. 159, 757 (2002)

8. Cao, J: A note on generalized q-difference equations for $q$-beta and Andrews-Askey integral. J. Math. Anal. Appl. 412, 841-851 (2014)

9. Carlitz, L: Generating functions for certain q-orthogonal polynomials. Collect. Math. 23(2), 91-104 (1972)

10. Fang, J-P: q-Differential operator identities and applications. J. Math. Anal. Appl. 332, 1393-1407 (2007)

11. Fang, J-P: Some applications of q-differential. J. Korean Math. Soc. 47, 223-233 (2010)

12. Fang, J-P: Note on a q-contour integral formula. Appl. Math. Comput. 233, 292-297 (2014)

13. Fine, NJ: Basic Hypergeometric Series and Applications. Mathematical Surveys and Monographs, vol. 27. Am. Math. Soc., Providence (1988)

14. Gasper, G, Rahman, M: q-Extensions of Barnes', Cauchy's, and Euler's beta integrals. In: Rassias, YM (ed.) Topics in Mathematical Analysis, pp. 294-314. World Scientific, Singapore (1989)

15. Gasper, G, Rahman, M: Basic Hypergeometric Series, 2nd edn. Cambridge University Press, Cambridge (2004)

16. Ismail, MEH, Masson, DR: q-Hermite polynomials, biorthogonal rational functions, and q-beta integrals. Trans. Am. Math. Soc. 346, 63-116 (1994)

17. Ismail, MEH, Stanton, D, Viennot, G: The combinatorics of $q$-Hermite polynomials and the Askey-Wilson integral. Eur. J. Comb. 8(4), 379-392 (1987)

18. Jackson, FH: On a q-definite integrals. Q. J. Pure Appl. Math. 41, 193-203 (1910)

19. Liu, Z-G: Some operator identities and q-series transformation formulas. Discrete Math. 265, 119-139 (2003)

20. Liu, Z-G: Two q-difference equations and q-operator identities. J. Differ. Equ. Appl. 16, 1293-1307 (2010)

21. Liu, Z-G: An extension of the non-terminating ${ }_{6} \phi_{5}$ summation and the Askey-Wilson polynomials. J. Differ. Equ. Appl. 17, 1401-1411 (2011)

22. Lu, D-Q: q-Difference equation and the Cauchy operator identities. J. Math. Anal. Appl. 359, 265-274 (2009)

23. Medema, JC, Álvarez-Nodarsea, R, Marcellán, F: On the q-polynomials: a distributional study. J. Comput. Appl. Math. $135,157-196$ (2001)

24. Rogers, L: On the expansion of some infinite products. Proc. Lond. Math. Soc. 24(1), 337-352 (1893)

25. Taylor, J: Several Complex Variables with Connections to Algebraic Geometry and Lie Groups. Graduate Studies in Mathematics, vol. 46. Am. Math. Soc., Providence (2002)

26. Wang, M-J: A remark on Andrews-Askey integral. J. Math. Anal. Appl. 341, 1487-1494 (2008)

27. Wang, M-J: A recurring q-integral formula. Appl. Math. Lett. 23, 256-260 (2010)

28. Range, RM: Complex analysis: a brief tour into higher dimensions. Am. Math. Mon. 110, 89-108 (2003) 
10.1186/1687-1847-2014-267

Cite this article as: Fang: Applications of a generalized $q$-difference equation. Advances in Difference Equations 2014, 2014:267

Submit your manuscript to a SpringerOpen ${ }^{\circ}$ journal and benefit from:

- Convenient online submission

- Rigorous peer review

- Immediate publication on acceptance

- Open access: articles freely available online

- High visibility within the field

- Retaining the copyright to your article

Submit your next manuscript at $\gg$ springeropen.com 